\title{
Reliable and Efficient Data Dissemination Protocol for Wireless Sensor Networks
}

\author{
Rohit Kumar Vishwkarma \\ Research Fellow \\ Dept. of computer science \\ Lovely professional university
}

\author{
Arvind Kumar \\ Astt. Professor \\ Dept. of computer science \\ Lovely professional university
}

\author{
Sitender Kumar \\ Research Fellow \\ Dept. of computer science \\ Lovely professional university
}

\begin{abstract}
Energy conservation plays a crucial in wireless sensor networks since such networks are designed to be placed in hostile and non-accessible areas where human presence is absolutely negligible. While battery-driven sensors will run out of battery sooner or later so this may responsible to stop working sensor network, the use of renewable energy sources such as solar power or gravitation may extend the lifetime of a sensor network. Proposed scheme utilize balancing the solar power and battery power in wireless sensor networks that can work unattended without replacing the battery sooner, this may increase the lifetime of the sensor network.
\end{abstract}

Keywords-wireless sensor network, clustering, energyefficiency, data dissemination, solar power.

\section{INTRODUCTION}

A wireless sensor network is a collection of nodes organized in to a cooperative network [13]. Each node consists of processing capability (one or more microcontrollers, CPUs or DSP chips) it may contain multiple types of memory like (program, data and flash memories), and have a RF transceiver (usually with a single omnidirectional antenna), have a power source (e.g., batteries and solar cells), and has various sensors and Actuators. The nodes communicate to each other wirelessly and often self-organize after being deployed in an ad hoc fashion. Wireless sensor networks are beginning to be deployed at an accelerated space. It is not unreasonable to expect that in 10-15 years that the world will be covered with wireless sensor networks with access to them via the Internet. This new technology is used to monitor unlimited numerous application areas including Habitat and Ecosystem Monitoring Automated Building Climate Control Wildfire detection various temperature measurements are Collected to produce a temperature map, Smart Parking, Security of Intra-Car, Event Detection, Structural Health Monitoring. The wireless sensor network (WSN) enables easy construction of such a large monitoring system because of the flexibility of wireless communication technology. However, in order not to restrict the advantages of the WSN, each sensor node usually has a battery for its energy source. This might be problematic in that dead battery replacement then it required for a huge number of WSN devices. Therefore, various energy saving techniques, including energyefficient routing protocols [8], medium access control schemes [9][10], special operating systems [11] and system-on chip technology [12], have been proposed.
Energy harvesting power sources

Depending on the application and location, there are four potential sources available for energy harvesting: light, vibration, heat, and RF. We'll discuss light in turn.

The remaining parts of the paper are organized as follow: Section 2 describes related work; Section 3 describes solar harvesting design; Section 4 deals with proposed scheme; Section 5 describes Result analysis; Section 6 Conclusions and future work; section 7 describes References.

\section{RELATED WORK}

J.Yang (2010)et alproposed an efficient data gathering algorithm in this protocol network grouped in to the cluster (each has a cluster head) and the nodes form chain in each cluster it checks for remaining energy to become a cluster head the cluster head adopts ant colony optimization to schedule access sequence of node EDGA can reduce energy consumption efficiently and increase life time of the sensor network[14]

M.minamiet al have proposed a battery less wireless sensor network for Environmental Monitoring Application so that battery replacement problem can be solved. In this paper author proposed schemes that how can we design the battery less wireless sensor network that uses the concept of combination of electric double layer capacitor with small solar cell solar cell work as an energy source in this case energy which is obtain form solar cell is very less so the sensor node has to wait until and unless it is sufficiently charged to communicate here sensor node has to wait for a very longer time so that is can be charge sufficiently after charging it work for very small amount of time so here author designing a mechanism that fits in to this practical application.[1]

M.Leeet al (2007) In this paper author has proposed a data dissemination schemes that will minimize the controloverhead In the context of wireless sensor network has different characteristics than the ad-hoc network, the main difference between wireless sensor network and ad-hoc networks is in the communication pattern used, and sensor network deals like (limited computing capability limited battery power low bandwidth and sensor node has to collect the data from different fixed location like from source to base station that may or may not be mobile)Routing algorithms are designed for general mobile ad-hoc network which can't be directly applicable to the wireless sensor network if we talk about one possible schemes for wireless sensor network each node maintain 
its hop distance and next hop nodes to the base station (or we can say multiple base station) so in this type of method it may be possible that it produce too much control overhead so to maintain the up-to-date and consistent hopdistance and next-hop nodes for all the sensor node which will present in the network at that time so after observing this overhead we propose a new low control -overhead data disseminations scheme, we referred to as pseudodistance data dissemination for efficiently disseminating the data packet for all sensor nodes to base station in the wireless sensor network. Wireless sensor network are made up of very low cost battery-powered sensor nodes with the wireless communication capability wireless sensor network sense environmental condition like humidity, temperature, sound, light, motion etc. [2]

R.K.Sahuet al (2012) in this paper author proposed a scheme of data dissemination in the wireless sensor network data dissemination to the multiple base-stations consume more energy. Many data dissemination schemes prosed for the data dissemination over the year to minimize the energy consumption in wireless sensor network in this paper author proposed a virtual infrastructure based data dissemination scheme which reduce the energy consumption in grid formation process Grid formation process is initiated by the source present in the sensor field when no valid Grid is available. Rest source available during the valid Grid is available all other sources available during the valid Grid time period and the share existing Grid. This scheme actually provides solution to calculate the cell size of the Grid and it handles different base-station in wireless sensor network. In this paper author has also describe the energy-efficient scheme to handle dissemination node failure. Wireless sensor network generally deals with base-station, event, and a large number of sensor nodes. Sensor nodes are very low cost and multifunctional device. Sensor nodes are randomly distributive over the large area and nodes are self-organize a large wireless sensor network. Sensor nodes monitor the event like humidity, temperature, heat, sound, presence of any object. If nodes sense any event happens around them, then sensor node produce some data and make the announcement to the base-station subscribing the data sensor node denote a source node and this is called data dissemination. [3]

\section{SOLAR HARVESTING DESIGN}

Here we are discussing about few component of solar energy harvesting, considering that best for its efficiency like embedded system which consume less energy (Few tens of $\mathrm{mW}$ ).

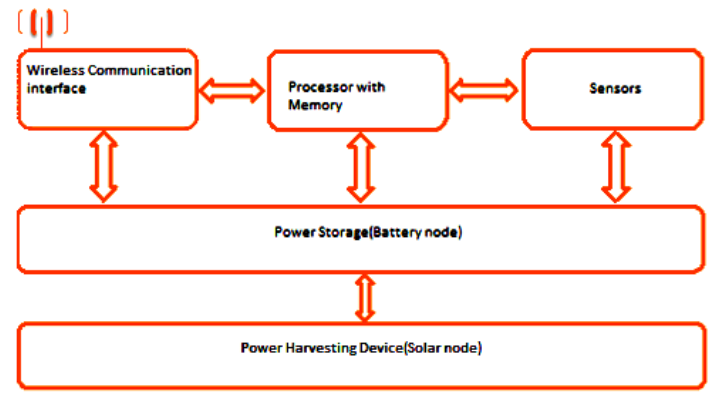

Fig 1solar harvesting design
Solar cells have different characteristics from battery, solar panel behaves as a voltage limited current source,there are optimal operating point at which solar cell charging maximize at that time.

\section{Energy storage}

There are two choices available for storing the energy

- Battery

- Electrochemical double layer capacitors

Batteries are good technology and have a higher energy density (more capacity for a given volume/weight) than capacitor but capacitor has higher power density than batteries now a day's capacitor available which are more energy-efficient than battery and have higher lifetime

\section{Harvesting circuit design}

Discussing about harvesting techniques the main part of harvesting module is harvesting circuit, which draw power from solar panels, and manage energy storage this circuit is design in such a way that maximize the efficiency.

\section{Energy measurement}

Module must have the energy measurement capacitor like low power battery monitor ICs will be providing this feature.

Table 1. Estimated levels of available ambient energy from the light sources

\begin{tabular}{|l|l|}
\hline Energy Source (Light) & Harvested Power Levels \\
\hline Indoor & $10 \mathrm{uW} / \mathrm{cm}^{2}$ \\
\hline Outdoor & $10 \mathrm{~mW} / \mathrm{cm}^{2}$ \\
\hline
\end{tabular}

\section{PROPOSED SCHEME}

In this paper we are discussing that how we can design our sensor network energy -efficient. Here we have proposed a scheme which operates on the $\mathrm{S}_{\mathrm{N}}$ and $\mathrm{N}_{\mathrm{N}}$.

\subsection{Assumption}

1 ID of the sensor node and their position are fixed and known to sensor itself and $\mathrm{CH}$ as well

2 All the sensor node $\left(S_{N}\right)$ has the same sensing range

3 All the sensor has same transmission range (d)

4 All the sensor consume same amount of energy

\subsection{Network Establishment phase}

In this section assumen nodes are uniformly distributes in a network and nodes longest transmission range is d.Fo clustered topology, $\mathrm{k}$ nodes are selected to be $\mathrm{CHs}$. Therefore each cluster has one $\mathrm{CH}$ and on average $(\mathrm{n}-\mathrm{k}) / \mathrm{k}$ CMs.

Section A Inthis section deploys $\mathrm{N}_{\mathrm{N}}$ and $\mathrm{S}_{\mathrm{N}}$ in the ratio of 30:70.And they are uniformly distributive in the region.

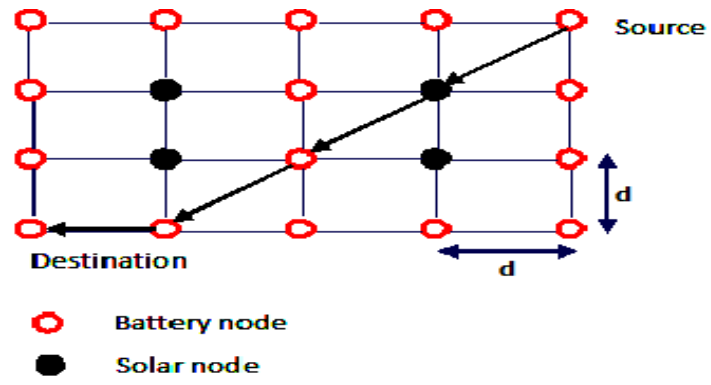

Fig 2Network Establishment 
Section B Inthe earlier protocol cluster head is selected very frequently in round figure so more energy wastage byperforming re-clustering in every round so.In this section initially define dedicated $\mathrm{N}_{\mathrm{N}}$ as a cluster head $(\mathrm{CH})$ (as we can see in above figure 2)based on their node ID And rest of the node $\left(\mathrm{S}_{\mathrm{N}}\right)$ act as $\mathrm{CM}$ until the $\mathrm{N}_{\mathrm{N}}$ energy level reached to some threshold value (assumed to be $30 \%$ of total energy) than further cluster headwill be selected among the $\mathrm{S}_{\mathrm{N}}$ when $\mathrm{N}_{\mathrm{N}}$ charge itself fully than again cluster head selected among the $\mathrm{N}_{\mathrm{N}}$ due to dedicated cluster head selection scheme it reduces energy consumption by not performing re-clustering in every round and due to $\mathrm{N}_{\mathrm{N}}$ it will increase lifetime of the sensor network, when source detect any event it aggregate sense data and send to the destination nodeby calculating shortest path based on the routing algorithm.

\subsection{Target area aware data dissemination}

In this case if the user (army man) wants to know what is happening in the particular location, in this case the query is forwarded towards the source location (where enemy reside) when request is reaches to the source location it will be flooded to all $\mathrm{N}_{\mathrm{N}}$ in this sub location As we can see

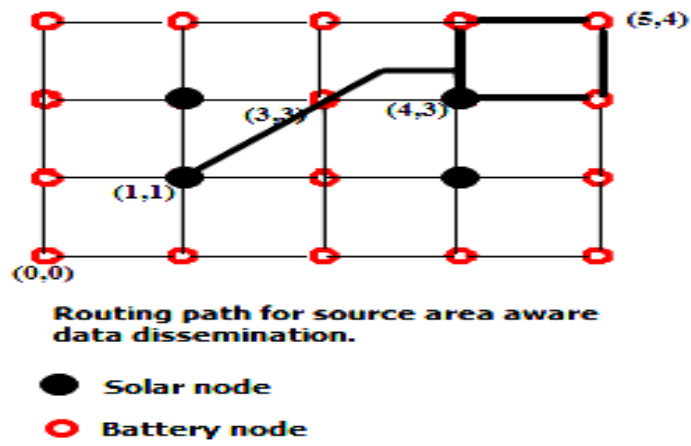

Fig 3 Data dissemination

in the above Figure 3 the army man in $(1,1)$ want to know the information of enemy in the region of $(4,3)(5,4)$ the query will be forwarded by diagonal path grid $(1,1)(3,3)$ and information pass forward $(4,3)(5,4)$ using horizontal path at the desired target location $(4,3)(5,4)$ and this query will be flooded toward all the neighbour node, here only $\mathrm{N}_{\mathrm{N}}$ involved in the flooding and $\mathrm{S}_{\mathrm{N}}$ participate in the forwarding process. This will reduce the energy consumption. A sensor node that can be in sleep mode and can later ask the on-going queries from the $\mathrm{N}_{\mathrm{N}}$ all the sensor node in the location will be aware that what kind of event they need to detect. In this case each active node retain a copy of information the $\mathrm{N}_{\mathrm{N}}$ will send re-election request again when detecting that a new node become active at that point of time if $\mathrm{N}_{\mathrm{N}}$ reached the level of $30 \%$ energy, then $\mathrm{CH}$ re-election process will be among the $\mathrm{N}_{\mathrm{s}}$

\subsection{Energy consumption model}

\begin{tabular}{|l|l|}
\hline Parameter Name & Value \\
\hline $\begin{array}{l}\text { Number of the sensor nodes ( N } \\
\text { ) }\end{array}$ & 100 \\
\hline Length of the packet ( k ) & 6bit \\
\hline $\begin{array}{l}\text { Initial energy of the sensor } \\
\text { nodes ( init E ) }\end{array}$ & $0-2 \mathrm{~J}$ \\
\hline
\end{tabular}

\begin{tabular}{|l|l|}
\hline $\begin{array}{l}\text { Energy consumption on circuit ( } \\
\text { elec E ) }\end{array}$ & $50 \mathrm{~nJ} / \mathrm{bit}$ \\
\hline $\begin{array}{l}\text { Channel parameter in free-space } \\
\text { model ( fs ) }\end{array}$ & $10 \mathrm{pJ} / \mathrm{bit} / \mathrm{m}^{2}$ \\
\hline $\begin{array}{l}\text { Channel parameter in multi-path } \\
\text { model ( Mp ) }\end{array}$ & $\begin{array}{l}0.0013 \mathrm{pJ} / \mathrm{bit} \\
/ \mathrm{m}^{4}\end{array}$ \\
\hline Distance between nodes(d) & $\mathrm{d}$ \\
\hline
\end{tabular}

As in [4] and [5],

let $\operatorname{ETx}(\mathrm{k}, \mathrm{d})$ the energy consumed to transmit a $k$ bits message over a distance $\mathrm{d}[6]$

$\mathrm{ETx}(\mathrm{k}, \mathrm{d})=$ Eelec $* \mathrm{k}+$ "amp $* \mathrm{k} * \mathrm{~d} 2$

Let $\mathrm{ERx}$ the energy consumed to receive a $k$ bits message:

$\operatorname{ERx}(\mathrm{k}, \mathrm{d})=\mathrm{ERx}-\operatorname{elec}(\mathrm{k})=$ Eelec $* \mathrm{k}$

Eelec $=50 \mathrm{~nJ} / \mathrm{bit}$ and $"=100 \mathrm{pJ} / \mathrm{bit} / \mathrm{m}^{2}$

The energy consumed by a sensor Si in Active/Sleep modes is calculated following the model proposed by [7]

ERadio $(\mathrm{Si})=$ PActive $*$ TActive + PSleep $*$ TSleep.....(4)

As in [7], PActive $=1040 \mathrm{~mW}$ and PSleep $=200 \mathrm{~mW}$.

There are some Notations that I will be using in this papersolar node $-\mathrm{N}_{\mathrm{N}}$, Sensor node- $\mathrm{S}_{\mathrm{N}}$,Distance $-\mathrm{dSolar}$ node energy- $\mathrm{EN}_{\mathrm{N}}$,Cluster head $-\mathrm{CH}$.

\section{Cluster-head selection algorithm}

Step 1Initially $\mathrm{N}_{\mathrm{N}}$ has $100 \%$ energy

Step 2set average minimum threshold of $\mathrm{N}_{\mathrm{N}}$

Step 3 if $\mathrm{N}_{N}<=$ minimum threshold

goto step 5

And charging start

Step 4 thenfind $\mathrm{CH}$ (Among the $\mathrm{S}_{\mathrm{N}}$ )

Step 5 sleep mode

$\mathrm{CM}$-Cluster member, $\mathrm{T}_{\mathrm{ac}}$-Active duration of the source node, $\mathrm{T}_{\mathrm{rem}}-$ Remaining time, $\mathrm{E}_{\mathrm{rem}}-$ Remaining Energy, $\mathrm{E}_{\text {min }}{ }^{-}$ Minimum Energy for transmission

\section{Energy balancing scheme}

Step 1 when (data sense)

Sense $\mathrm{CM}($ sink $) \quad \mathrm{n}=1,2,3 \ldots$

Else

goto step 5

Step 2 if (CM=wakeup)

Gather CMdetails such as

$\mathrm{CM}_{=}\left(\mathrm{T}_{\text {rem }}, \mathrm{T}_{\mathrm{ac}}, \mathrm{E}_{\mathrm{rem}}, \mathrm{E}_{\mathrm{min},}\right.$ Node $\left.\mathrm{ID}_{\mathrm{i}}\right)$

Where $i=1,2,3,4 \ldots$

Step 3 if $\left(\left(\mathrm{T}_{\mathrm{rem}}>\mathrm{T}_{\mathrm{ac}}\right) \& \&\left(\mathrm{E}_{\mathrm{rem}}>\mathrm{E}_{\mathrm{min}}\right)\right)$

Establish connection using routing protocol

goto step 4

Else

goto step 5

Step 4 continue transmission

Step 5 goto sleep mode

\section{RESULT ANALYSIS}

The below figure shows that how solar node will be charging itself and in what duration, there are four solar nodes which will be charging accordingly 


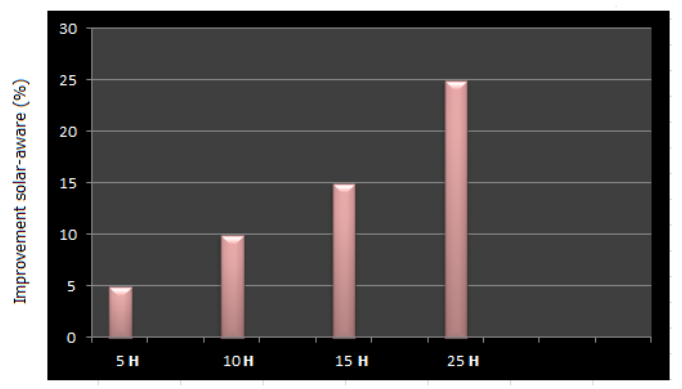

Number of sunnodes

Fig 4

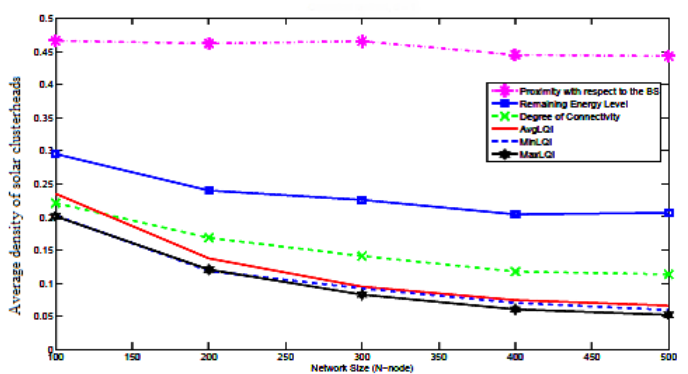

Fig 5: Average density of solar cluster heads

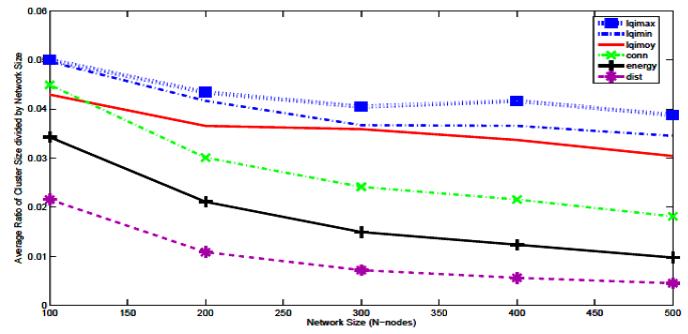

Fig 6 : Average cluster sizes divided by network size

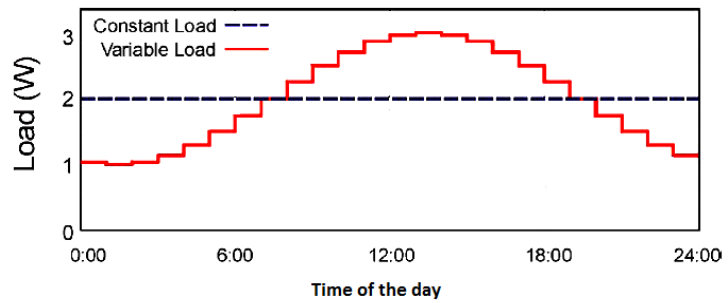

Fig 7:Example of two $50 \mathrm{Wh}$ per day load profiles.

\section{CONCLUSIONS AND FUTURE WORK}

Environmental energy harvesting has recently emerged as a viable option to supplement battery supplies in energy constrained embedded systems. However, designing an efficient solar harvesting systeminvolves an understanding of several factors. This paper systematically analyzed the various components, design choices, and tradeoffsinvolved in the design of a solar energy harvesting module and their impact on the efficiency. The Proposed schemes show how harvesting aware power management improves energy usage compared to only battery aware approaches. Proposed schemes shows the design and performance evaluation of $\mathrm{N}_{\mathrm{N}}$ and $\mathrm{S}_{\mathrm{N}}$ nodes, experimental results indicate the feasibility of near perpetual operation of harvesting aware, outdoor sensor networks and efficiently reduce the energy consumption and it increase network lifetime as well.

\section{REFERENCES}

[1] Masateru MINAMI, Takashi MORITO, Hiroyuki MORIKAWA and Tomonori AOYAMA Solar Biscuit: A Battery-less Wireless Sensor Network System for Environmental Monitoring Applications

[2] Min-Gu Lee, Sunggu Lee, Data Dissemination for wireless Sensor Network 2007.

[3] Ravi Kant Sahu, AbhishekMohta, L.K Awasthi. Energy-efficient Grid-Based Data Dissemination in Wireless sensor Network.

[4] C. Diallo, A. Gupta, M. Becker, and M. Marot. Energy aware database updating protocols for auto configurable sensor networks. In Globe Net 2009, the 8th international conference on Networks, ICN'09, Cancun, Mexico, Mar.2009.

[5] C. Diallo, A. Gupta, M. Marot, and M. Becker. Virtual base station election for wireless sensor networks. In ACM Notere 2008, the $8^{\text {th }}$ international conference on New Technologies in Distributed Systems, Vol. 2, Lyon, France, Jun. 2008.

[6] W. B. Heinzelman, A. Chandrakasan, and H. Balakrishnan.An application-specific protocol architecture for wireless microsensor networks.IEEE Transactions on Wireless Communications, Vol. 1(4), pages660-670, Oct. 2002.

[7] E. Shih, S.-H. Cho, N. Ickes, R. Min, A. Sinha, A. Wang, and A. Chandrakasan. Physical layer driven protocol and algorithm design for energy-efficient wireless sensor networks. In ACM MobiCom, Rome, Jul. 2001.

[8] Ian F. Akyildiz,W. Su, Y. Sankarasubramaniam and E. Cayirc, "A survey on Sensor Networks", IEEE Communications Magazine, Vol. 40, No. 8, pp.102114, 2002.

[9] W. Ye, J. Heidemann and D. Estrin, "An EnergyEfficient MAC Protocol for Wireless Sensor Networks", In Proceedings of the 21st International Annual Joint Conference of the IEEE Computer and CommunicationsSocieties (INFOCOM2002), New York, NY, USA, June 2002.

[10] T. van Dam and K. Langendoen, "An Adaptive Energy- Efficient MAC Protocol for Wireless Sensor Networks", In Proceedings of the 1st ACM Conference on Embedded Networked Sensor Systems, Los Angeles, CA, USA, November 2003.

[11] J. Hill, R. Szewcyzk, A. Woo, S. Hollar, D. Culler, and K. S. J. Pister, "System architecture directions for networkedsensors", Proceedings of the 9th International Conference on Architectural Support for Programming Languages and Operating Systems (ASPLOS), November 2000

[12] J. Rabaey, J. Ammer, J. L. da Silva Jr., D. Patel, and S. Roundy, "Pico Radio Supports Ad Hoc Ultra-Low Power Wireless Networking," IEEE Computer, vol. 33, no. 7, pp. 42-48, July 2000.

[13] J. Hill, R. Szewczyk, A, Woo, S. Hollar, D. Culler, and K. Pister, System Architecture Directions for Networked Sensors, ASPLOS, November 2000.

[14] Jing Yang and Zetao Li Yi Lin Wei Zhao: A Novel Energy-Efficient Data Gathering Algorithm for Wireless Sensor Networks 2010. 